\title{
Parameter-correlation study on shock-shock interaction using a machine learning method
}

\author{
Peng J. ${ }^{\mathrm{a}, \mathrm{b}}$, Luo C.T. ${ }^{\mathrm{a}}$, Han Z.J. ${ }^{\mathrm{a}, \mathrm{b}}$, Hu Z.M. ${ }^{\mathrm{a}, \mathrm{b}, *}$, Han G.L. ${ }^{\mathrm{a}, \mathrm{b}}$, Jiang Z.L. ${ }^{\mathrm{a}, \mathrm{b}}$ \\ a State Key Laboratory of High-temperature Gas Dynamics, Institute of Mechanics, Chinese Academy of Sciences, Beijing 100190, China \\ b School of Engineering Science, University of Chinese Academy of Sciences, Beijing 100049, China
}

\section{A R T I C L E I N F O}

\section{Article history:}

Received 30 June 2020

Received in revised form 8 September 2020

Accepted 26 September 2020

Available online 7 October 2020

Communicated by Cheng Wang

\section{Keywords:}

Shock-shock interaction

Machine learning

Hypersonic flow

Impinging jet

Genetic programming

Triple point

\begin{abstract}
A B S T R A C T
To predict the maximum heating load induced by shock-shock interaction more reliably and accurately, the geometrical scale of the overall wave configuration of shock-shock interaction is very useful. However, it is hard to be solved with traditional shock theory due to its complexity. The results of numerical and experimental studies are case-by-case. Concise formulas correlating the geometrical scales of shock-shock interaction with the given flow parameters are desired but still unavailable. In the present work, a set of correlative formulas for the triple-points' coordinates of type IVa, IV, and III shock-shock interaction are derived by multilevel block building algorithm, a functional machine learning method. The key flow structure of shock-shock interaction, i.e., the supersonic impinging jet, can be determined with the help of shock theories and the formulas. In addition, the transition criteria respectively for the overall wave configuration transitions of type IVa $\leftrightarrow$ type IV and type IV $\leftrightarrow$ type III shock-shock interaction can be obtained by the machine learning based method.
\end{abstract}

(c) 2020 Elsevier Masson SAS. All rights reserved.

\section{Introduction}

Shock-shock interaction (SSI) is a classical phenomenon in hypersonic flows. It performs complex flow structures and can induce a tiny region with extremely high pressure and heating loads when type IV shock-shock interaction occurs. It may form a supersonic jet impinging directly upon the body surface, where the heating load might be ten times larger than that in the area slightly away from the impinging point. This would be a great challenge to the thermal protection of the vehicle. As for the engineering design, a quick way to acquire the magnitude and location of the maximum heating load is urgently needed. Such an extremum mainly depends on the jet impinging location and its width. However, the two key factors are hard to get systematically due to the lack of theoretical solutions to the overall configuration of the SSI. In this paper, we use machine learning to solve this problem.

Shock-shock interaction has attracted a lot of attention since the 1950s. Edney [1] is the first one to scientifically investigate the SSI between oblique and bow shock waves. He classified the shock-shock interactions into six types by the interacting pattern.

\footnotetext{
* Corresponding author.

E-mail address: huzm@imech.ac.cn (Z.M. Hu).
}

The overall interaction configurations alternate with the shock intersecting point moving around the circumference of the cylinder. Hereinto, the type IV interaction occurs when the slip layers of triple points form a thin supersonic jet impinging upon the surface. It has been studied largely for the unique supersonic jet flow and the extraordinary heating load. Keyes [2] used the oblique shock relations and Prandtl-Meyer relations to solve the flow parameters and predict the pressure peak and the heat transfer rate. He also pointed out that type III and IV interactions would cause the most serious heating load. Holden and Wieting [3,4]experimented with the oblique shock wave interacting with the bow shock wave over a cylinder. They measured the distribution of heat transfer and pressure in the peak heating regions around the cylinder. In addition, many numerical studies [5-8] reported the unsteady characteristics of the SSI, the interaction of shear-layers within the shock-layer, and acoustical feedback between the bow shock and the body. Furumoto [9] studied the real-gas effects on the hypersonic shock-wave/boundary-layer interaction and found that type IV shock-shock interference heating flows with real-gas effects are inherently unsteady. Serge [10] performed an experiment of SSI in high Mach number flows and revealed an influence of incoming disturbances on the stagnation line heat transfer. Xiao [11] studied the SSI on a blunt body with a forward-facing cavity and found that the flow could be either quasi-steady or unsteady, depending on the supersonic jet impingement location. Khatta [12] experimentally investigated the interaction between oblique shock 


\section{Nomenclature}

SSI shock-shock interaction

IS incident oblique shock

BS bow shock

SL shear layer

TS transmitted shock

NS a normal shock

DL deep learning

SR symbolic regression

GP genetic programming

BBP block building programming

MBB multilevel block building algorithm

HLLC Harten-Lax-van Leer contact

Ma Mach number

$\beta \quad$ the angle of the incident oblique shock

Ir non-dimensional location parameter
P pressure

$\mathrm{T}$ temperature

$\gamma \quad$ specific heat ratio

$\mathrm{R} \quad$ radius of the cylinder

$\mathrm{N}_{r} \quad$ number of radial grids

$\mathrm{N}_{\varphi} \quad$ number of circumferential grids

$L \quad$ the distance of the upper bow shock to the wall

$r \quad$ the distance of triple point A to the cylinder center

Subscripts

A the triple point $A$

B the triple point $B$

$\infty \quad$ the free stream

$\mathrm{w}$ the wall and bow shock of a hemisphere and provided evidence of the unsteady/non-steady nature inherent to the SSI phenomenon.

However, to the authors' knowledge, only two reports have estimated the shock geometry for the most interesting shock-shock interactions, type IV and III. In 2003, Grasso [13] reported that the most critical conditions occurred when the supersonic jet was nearly perpendicular to the body surface. In the report, he described the shape of the detached bow shock in SSI with the hyperbola formula given by Billig [14] for a bow shock wave without oblique shock interaction. Then he characterized the criterion for the type transition and estimated the aerothermal loads. However, the SSI would change the detached distance of the bow shock greatly, especially when type III and IV occurred. Therefore, such a shape assumption of bow shock might be unreliable. In 1997, Frame [15] reported a theoretical model to derive the length of the transmitted shock and jet geometry by calculating the shape of the bow shocks using a continuity methodology. However, too many simplifications in this theoretical model might affect its precision and the applied iterating algorithm is very complex to use by others. In the aforementioned studies, the proposed theoretical methods both have shortcomings. The reason why the shape of the detached bow shock of the SSI structure is hard to calculate is that the shape of the bow shock and the location of the jet impinging the body surface affect each other. The standoff distance of the bow shock in an oblique-bow shock-shock interaction configuration is different from that of a bow shock alone without interaction. It is affected by the two downstream subsonic regions separated by the supersonic jet flow. However, the location and geometry of the jet also depend on the shock intersecting location that is related to the standoff distance of the bow shock. The subsonic region can transmit the message upstream from the body to the bow shock, which makes the feedback mechanism possible. That is the reason why it is hard to estimate the shock geometry.

In recent years, the development of machine learning has made a lot of the impossible possible. Machine learning came to the forefront when AlphaGo defeated the best human go player. It is widely used in many fields, especially successful in image and speech recognition. It was also applied in fluid mechanics lately. Allison [16] presents a machine learning approach to wind velocity estimation based on quadcopter state measurements without a wind sensor. Yan [17] applied a machine learning technique in the aerodynamic shape optimization and significantly decreased the required CFD calls by over 62.5\%. Yu [18] used machine learning to replace the aircraft dynamics simulation and found it could significantly improve prediction performances and effectively reduce the training costs.
In this paper, we performed a lot of numerical simulations to get enough flow fields of shock interaction as the training samples. Then we chose a proper machine learning algorithm to obtain new formulas for shock intersecting points from these training samples. After a validation of the formulas we used them to predict the jet impinging location. In addition, we got a transition criteria of shock interaction types from the training samples with the developed method.

\section{Methods}

\subsection{Theory analysis of shock-shock interaction}

The type IV interaction presents the most complex shock structure and heating load (Fig. 1 (a)). The incident oblique shock (IS) intersects with the upper bow shock (BS1) induced by the cylinder at point A producing a shear layer (SL1) and a transmitted shock (TS). The intersecting point of the three shock waves is called the upper triple point labeled by A. The TS intersects with the lower bow shock (BS2) at the lower triple point labeled by B producing a shear layer (SL2) and a reflected shock that intersects with the SL1 at point C. The overall wave configuration of shock-shock interaction features two triple points and the supersonic jet flow surrounded by two shear layers between the subsonic regions, (4) and (6). The shock wave (BC) is successively reflected from the shear layers SL1 and SL2 resulting in a series of expansion waves and compression waves within the jet. At last, the jet hits the surface of the cylinder directly and induces a normal shock (NS) and a tiny region with high pressure and heating loads along the surface. It might occur the shock wave/boundary layer interaction $[19,20]$. The standoff distance of NS in supersonic impinging jet is also an unresolved issue. This kind of problem has been studied by Lim [21]. The flow field of type IV interaction is primarily divided into seven regions which are labeled by numbers from (1) to (7) as schematically depicted in Fig. 1 (a). The flow parameters in the aforementioned regions can be theoretically solved by the oblique shock relations and Prandtl-Meyer relations when the condition of free stream and the shock angle $\beta$ are known. However, the actual sizes of the jet and TS remain unknown until the locations of triple points $A$ and $B$ are known.

When the oblique shock moves up, the jet will turn up and wipe along the surface of the cylinder, which forms type IVa shock-shock interaction (Fig. 1 (b)). When the oblique shock moves down, the SL1 will hit the surface of the cylinder without jet impingement, which forms type III shock-shock interaction (Fig. 1 (c)). For the aforementioned three types of shock-shock 


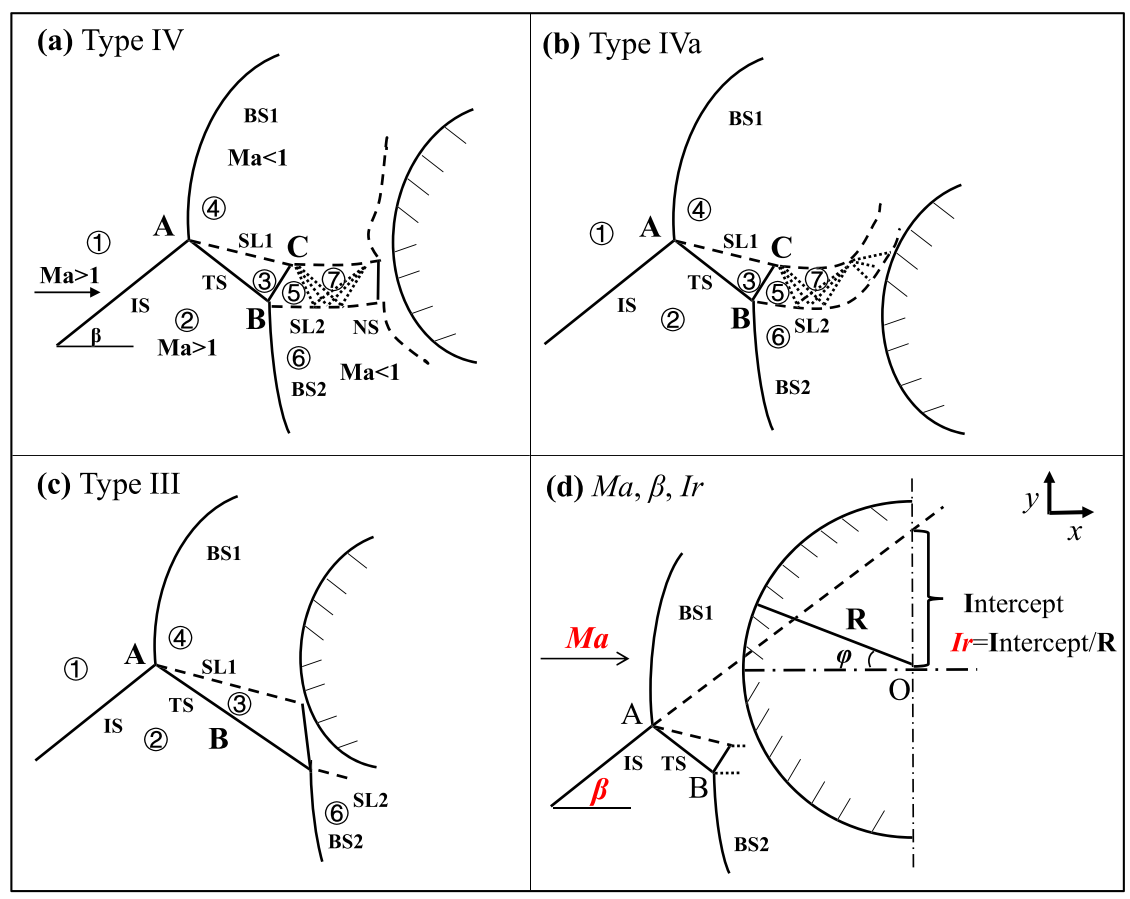

Fig. 1. The shock interacting patterns and three key factors.

interactions, there are three normalized factors mainly determining the shock geometry: the Mach number of the free stream $M a$, the incident shock angle $\beta$, and the location parameter Ir (Fig. 1 (d)). We defined $I r$ as the ratio of the intercept of the incident shock wave on the vertical axis to the cylinder radius $\mathrm{R}$. If $M a$ of the freestream flow and the shock angle $\beta$ are given, the state parameters in each flow region as labeled in Fig. 1 (a-c) can be theoretically obtained by solving the oblique shock relations. That is to say, Ir only affects the shock intersecting location and the scale of the overall wave structures rather than the flow state parameters. As for the configuration of SSI, when the three key factors are given, the coordinates of triple points $\mathrm{A}$ and $\mathrm{B}$ can be determined consequently. In this paper, we use the machine learning method to figure out the relationship between $\mathrm{x}$ coordinates of triple points and the three key factors $M a, \beta$, and $I r$

$x_{\mathrm{A}}=f_{1}(M a, \beta, I r), \quad x_{\mathrm{B}}=f_{2}(M a, \beta, I r)$.

According to the fact that triple point $\mathrm{A}$ is on the line of IS, we can get

$y_{\mathrm{A}}=\tan \beta \cdot x_{\mathrm{A}}+I r$.

Through the angle of TS acquired by solving the oblique shock relations, $\beta_{\mathrm{TS}}$, we can get

$y_{\mathrm{B}}=\tan \beta_{\mathrm{TS}} \cdot\left(x_{\mathrm{B}}-x_{\mathrm{A}}\right)+y_{\mathrm{A}}$.

In the same way, we can get the coordinates of point $C$ and the angles of SL1 and SL2. As a result, the starting boundary BC of the jet flow is known. Considering the deflecting effect of the expansion and compression waves, we can predict approximately the flow direction of the jet. At last, we can get the jet width and its impinging location for type IV interaction.

\subsection{Numerical method and grid validation}

Machine learning for aerodynamic problems needs sufficient data of samples which can be obtained by numerical simulations.
In the present work, the two-dimensional compressible NavierStokes equations are solved using the finite-volume method. The numerical flux through each cell face is evaluated using a secondorder TVD (total variation diminishing) scheme based on a nonlinear Riemann solver named HLLC (Harten-Lax-van Leer contact). The HLLC scheme can resolve the isolated shock and slip line exactly while remaining positively conservative [22]. The minmod limiter is used to suppress spurious oscillations near the discontinuities while high-order accuracy is retained away from the jumps. The temporal integration is performed with a second-order pointimplicit scheme. Considering the real-gas effect, the specific heat of air is modeled by a fourth-order fitting polynomial about temperature for which the fitting coefficients can be taken from the report [23]. This computational code has been used in the previous work and validated by Zhang [24] and Lu [25] for the study of hypersonic flows including the shock-shock interaction problems. In addition, because of the low accuracy of spatial discretization and poor grid, stochastic error [26] would accumulate with the time integration [27]. To get the accurate results, the grid independence study should be conducted carefully and will be discussed hereafter.

For the validation of the numerical method and the evaluation of grid independence, the experimental data of type IV interaction over a cylindrical test model in the reported work [4] was take to compare with the numerical results we performed with three different levels of grid. This experiment was always taken as the numerical validation case in literatures $[5,8,11,25]$. The test conditions of the case are listed in Table 1 while the details of the three grids are exhibited in Table 2. The distributions of mean pressure on the circumferential angle of the cylinder $\varphi$ are obtained respectively from the present simulations and the reported experiment. They are combined in Fig. 2 (a) while the comparison of numerical and experimental heat fluxes is depicted in Fig. 2 (b). The squares denote the experiment data while the curves are the numerical results. For the numerical heat flux, it was obtained based on the temperature at the middle of first two layers of grid close the wall and the wall temperature $\mathrm{T}_{\mathrm{w}}$. Tyurenkova et al. [28] proposed two methods to compute the heat flux on the wall and had made great progress in this field. Considering that the pres- 


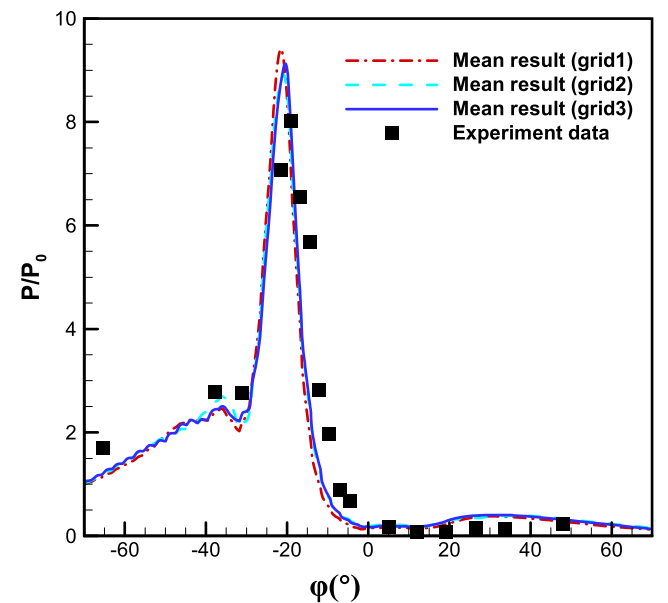

(a) Pressure

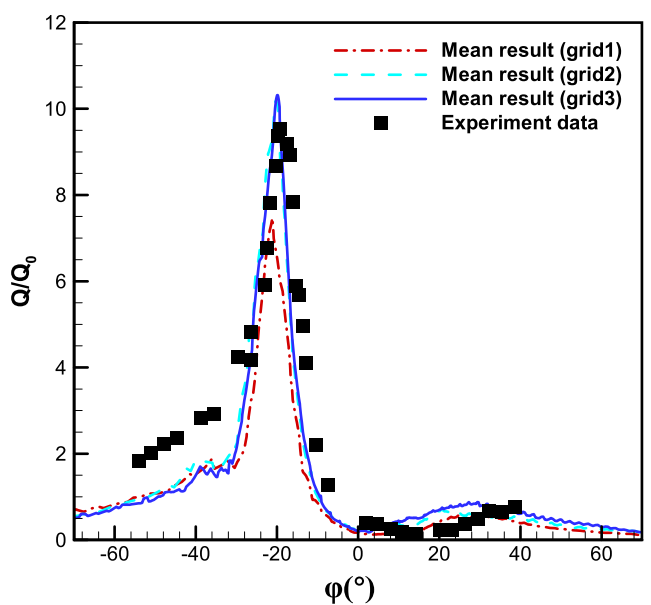

(b) Heat flux

Fig. 2. The comparison of the distribution of pressure and heat flux along the cylindrical model surface.

Table 1

The test conditions of the experiment on type IV shock-shock interaction [4].

\begin{tabular}{ll}
\hline Variables & Value \\
\hline Mach number $M a_{\infty}$ & 8.03 \\
Temperature $\mathrm{T}_{\infty}, \mathrm{K}$ & 111.56 \\
Pressure $\mathrm{P}_{\infty}, \mathrm{Pa}$ & 985.01 \\
Shock angle $\beta,^{\circ}$ & 18.1114 \\
Wall temperature $\mathrm{T}_{\mathrm{w}}, \mathrm{K}$ & 294.44 \\
Unit Reynolds number, ${ }^{-1}$ & $6.7 \times 10^{6}$ \\
Specific heat ratio $\gamma$ & 1.4 \\
Cylinder radius $\mathrm{R}, \mathrm{m}$ & 0.038 \\
\hline
\end{tabular}

Table 2

The details of three grids.

\begin{tabular}{llll}
\hline Grid & $\mathrm{N}_{r} \times \mathrm{N}_{\varphi}$ & Surface cell thickness, $\mathrm{m}$ & Grid stretching ratio \\
\hline Grid 1 & $200 \times 336$ & $1 \times 10^{-5}$ & 1.3 \\
Grid 2 & $500 \times 476$ & $1 \times 10^{-6}$ & 1.2 \\
Grid 3 & $600 \times 696$ & $1 \times 10^{-6}$ & 1.2 \\
\hline
\end{tabular}

sure and heat flux appear fluctuations with time on the cylindrical wall due to the unsteady characteristic of the SSI, we output the transient value every 10 computational steps. The transient value was varied with the time increase and so do their mean value. But over enough time, the mean value would converge to a fixed value and we used this fixed value as the mean value. Here, the mean pressure and heat flux are respectively normalized by the stagnation pressure $P_{0}$ and heating flux $Q_{0}$ at the stagnation point of the cylindrical model without oblique shock-shock interaction in the same free stream. The Mach number contours obtained by three grids are drawn in Fig. 3 for detailed comparison.

Just as mentioned above, the poor grid would make the stochastic errors accumulate and the refined grid is really needed. In Fig. 2 (a), the pressure distributions of numerical simulations almost coincide with the experiment data. In addition, the heat flux distributions obtained respectively with grid 2 and grid 3 both approximately agree with the experiment as can be seen from Fig. 2 (b) while grid 1 fails to capture the peak value. In the present study, we focus on the overall wave configuration of SSI. Therefore, we compared the locations of triple points A and B respectively simulated with three different grids in Fig. 3. We can see the shock configurations of different simulations almost overlap each other. Considering the failure in capturing peak heat flux of grid 1 and the unsteady characteristic of the SSI, we conclude that grid 2 and grid 3 are both acceptable for the present study. Since machine learning requires a large number of samples, we chose grid 2 to perform all the numerical simulations in the coming sections.

\subsection{Machine learning method}

Many machine learning methods have been proposed in the last decades. Among them, deep learning (DL) is the most popular one. It is very powerful because it has the ability to extract features automatically. However, the training of DL needs a large amount of data, and its target model involves many hidden coefficients. This means it is very expensive to get a reliable DL model. Even if the DL model is properly identified, its application is still inconvenient because it is a gray/black-box type model with a large number of coefficients, not to mention its questionable generalizability with the limited training set. In fact, every machine learning method has its own pros and cons. For our specific task, detecting concise formulas (as donated in Section 2.1) that can describe the configuration of shock-shock interaction with a limited number of data, it is obvious that DL does not work well.

Symbolic regression (SR) is a special machine learning technique (reinforcement learning). What sets it apart from other methods is its free-form expressions. It does not impose prior assumptions on the structure of the target model, and let the optimization process search potential formulas such that it best fits the training/validation data and be as concise as possible. Compared to deep learning, SR requires much less training data and can get concise models with explicit mathematical expressions. This makes SR a good candidate for modeling the overall wave configuration of shock-shock interaction.

Genetic programming (GP) is the most popular method for symbolic regression. The idea behind GP is to apply Darwin's theory of natural evolution, survival the fittest, to the artificial world of computers and modeling. Theoretically, GP can get the best result provided that the computation time is long enough. However, the convergence speed of GP could be very slow for large scale problems with a large number of variables. This limited its practical applications. To improve efficiency, a number of techniques have been proposed. State-of-the-art SR methods include block building programming (BBP) [29], evolutionary feature synthesis [30], multilevel block building (MBB) [31], and multiple regression genetic programming [32]. Luo et al. [33] has compared their performance on hypersonic aerodynamic data modeling, and find that MBB performs the best. In MBB, the target model is decomposed into several blocks and then into minimal blocks and factors (Fig. 4). $x_{1}, x_{2}$, and $x_{3}$ are independent variables, $\varphi(x)$ is the basic function, and $f(x)$ is the target function. The minimal factors 


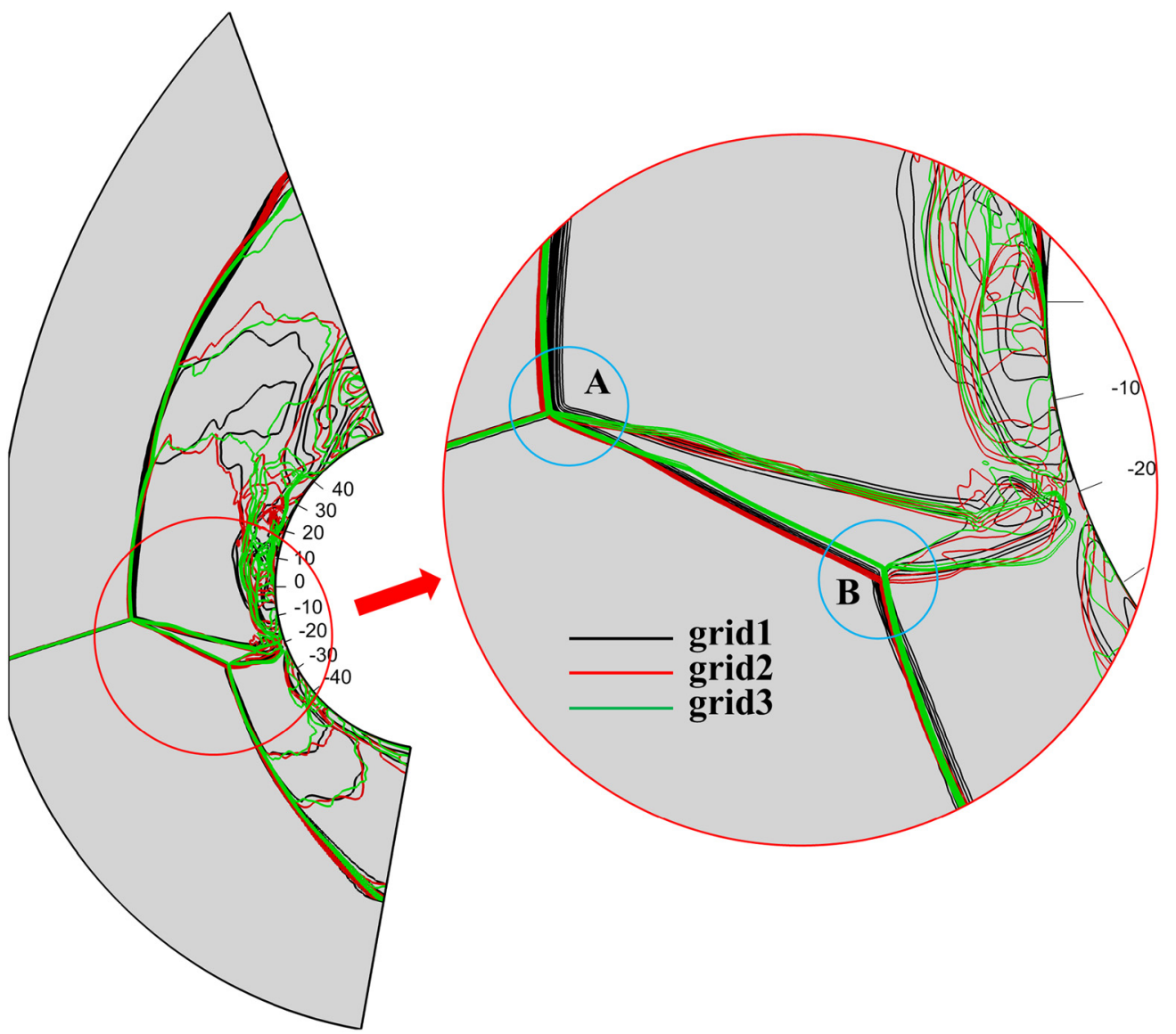

Fig. 3. Numerical contours of Mach number obtained with three grids.

$$
\begin{aligned}
& f\left(x_{1}, x_{2}, x_{3}, x_{4}, x_{5}, x_{6}\right) \\
& \text { Level-1 block search } \\
& f(\mathbf{x})=c_{1} * \frac{\varphi_{1}\left(x_{1}\right)+c_{2} * \varphi_{2}\left(x_{2}, x_{3}, x_{4}, x_{5}, x_{6}\right)}{\text { Level-2 block search } \quad \text { with variable } x_{5} \text { fixed }} \\
& f(\mathbf{x})=c_{1} * \frac{\varphi_{1}\left(x_{1}\right)+c_{2} * \varphi_{2}\left(x_{4}, x_{5}\right)+c_{3} * \varphi_{3}\left(x_{2}, x_{3}, x_{6}, x_{5}\right)}{\text { Level-3 block search } \quad \text { with variable combination }\left(x_{5}, x_{6}\right) \text { fixed }} \\
& f(\mathbf{x})=c_{1} * \varphi_{1}\left(x_{1}\right)+c_{2} * \varphi_{2}\left(x_{4}, x_{5}\right)+c_{3} * \varphi_{3}\left(x_{2}, x_{6}, x_{5}\right)+c_{4} * \varphi_{4}\left(x_{3}, x_{6}\right)
\end{aligned}
$$

Fig. 4. Illustration of MBB [31].

are relatively much easier to determine for most conventional GP or other non-evolutionary algorithms. Thus, MBB is more effective and efficient. Therefore, MBB is selected as the data modeling method in this study.

\section{Numerical and machine learning results}

\subsection{The numerical results and the correlation}

Series simulations of shock-shock interactions are conducted to get adequate sample data. The conditions of these cases are listed in Table 3. We choose the atmospheric parameters, e.g., the static pressure and temperature, at an altitude of $30 \mathrm{~km}$ as the free stream flow conditions. In addition, the range of the Mach number covers the hypersonic flight regime, i.e., M6 10. The shock angle $\beta$ and the location parameter $I r$ is also chosen in a suitable range for the type IV, IVa, and III interactions.

Totally, we conduct simulations of $5 \times 9 \times 5=225$ cases and record the coordinates of the triple points $\mathrm{A}$ and $\mathrm{B}\left(x_{\mathrm{A}}, y_{\mathrm{A}}, x_{\mathrm{B}}, y_{\mathrm{B}}\right)$ and the interaction types for each case. The simulation results indicate that the unsteady characteristic of the SSI concentrates on the free part of the supersonic jet flow which is surround by two shear layers and does not influence the triple point location distinctly. So, uncertainties induced by the unsteady behavior of the SSI to the present method are negligible. The data structure of the output is shown in Table 4 where ten cases out of 225 are listed 
Table 3

Cases of numerical simulation.

\begin{tabular}{ll}
\hline Variables & Value \\
\hline Pressure, $\mathrm{P} / \mathrm{Pa}$ & 428 \\
Temperature, $\mathrm{T} / \mathrm{K}$ & 200 \\
Mach number, $\mathrm{Ma}$ & $6,7,8,9,10$ \\
${\text { Shock angle, } \beta /^{\circ}}^{\circ}$ & $15,16,17,18,19,20,21,22,23$ \\
Location parameter, $I r$ & $0.3,0.4,0.5,0.6,0.7$ \\
\hline
\end{tabular}

\section{Table 4}

Data structure of the output of numerical simulations: coordinates of triple points and the interaction types.

\begin{tabular}{llllllll}
\hline$I r$ & $M a$ & $\beta /^{\circ}$ & $x_{\mathrm{A}}$ & $y_{\mathrm{A}}$ & $x_{\mathrm{B}}$ & $y_{\mathrm{B}}$ & Type \\
\hline$\ldots$ & $\ldots$ & $\ldots$ & $\ldots$ & $\ldots$ & $\ldots$ & $\ldots$ & $\ldots$ \\
0.7 & 6 & 19 & -1.8160 & 0.0881 & -1.5547 & -0.0772 & IVa \\
0.6 & 6 & 19 & -1.7832 & -0.0019 & -1.5048 & -0.1793 & IVa \\
0.5 & 6 & 19 & -1.7297 & -0.0845 & -1.4392 & -0.2754 & IVa \\
0.4 & 6 & 19 & -1.7346 & -0.1866 & -1.2131 & -0.5270 & IV \\
0.3 & 6 & 19 & -1.7528 & -0.2973 & -1.0404 & -0.7385 & III \\
0.7 & 6 & 20 & -1.8579 & 0.0347 & -1.5500 & -0.1407 & IVa \\
0.6 & 6 & 20 & -1.8151 & -0.0510 & -1.4991 & -0.2344 & IVa \\
0.5 & 6 & 20 & -1.7682 & -0.1340 & -1.4214 & -0.3442 & IV \\
0.4 & 6 & 20 & -1.7749 & -0.2371 & -1.1844 & -0.6093 & IV \\
0.3 & 6 & 20 & -1.7936 & -0.3455 & -0.9863 & -0.8061 & III \\
$\ldots$ & $\ldots$ & $\ldots$ & $\ldots$ & $\ldots$ & $\ldots$ & $\ldots$ & $\ldots$ \\
\hline
\end{tabular}

as examples. For concision, the output data for all the 225 computed cases are not given here. The coordinates in the table are normalized by the radius of the cylinder. In the record, most are type IV interaction and the rest are type IVa or III. When the Mach number and shock angle are constant, as shown in Fig. 5, with the decrease of Ir, the interaction type changes from IVa to IV and then to III successively. Furthermore, as described in Section 2.1, the flow state parameters such as the static pressure, the local flow Mach number, and the flow direction in each flow region as labeled in Fig. 1 are determined respectively if the shock angle and the Mach number of the freestream flow are given. Therefore, all the five transmitted shock waves, as labeled by TS or line segment $A B$ in Fig. 1, parallel to each other in each frame of Fig. 5. So do all the reflected shock waves as labeled by $\mathrm{BC}$ and all the slip layers as labeled by SL1 or AC in each frame. We can see that the five triangles consisted of points A, B, and C as depicted in Fig. 5 (a) or (b) are similar to each other. At the same time, in Fig. 5 (a), the upper triple points of three type IVa interactions $(I r=0.5,0.6,0.7)$ seem to be approximately in a straight line and those of the two type IV interactions ( $I r=0.3,0.4)$ are in a different straight line. It can also be seen in Fig. 5 (b) that the upper triple point of the type III interaction ( $I r=0.3$ ) seems to diverge from the approximately straight line including the upper triple points of the other four cases which are type IV interactions. Therefore, the relationship functions between the coordinates of the upper triple point $\mathrm{A}$ and $I r$ should be different for different interaction types, i.e., IVa, IV, and III, respectively. As the boundaries among the three interaction types cannot be determined and input to the machine learning process, combining three different functions into one will bring a challenge to the machine learning part. It seems that separating the data by the interaction type and getting the different formulas for the different interaction type might be a good idea. However, considering the application of these formulas, we always do not know the interaction type beforehand as only the three key factors $M a, \beta$ and $I r$ are given. It is difficult for us to choose which formula to use. Therefore, we think that general formulas would be better from the applying perspective.

With the output data of numerical simulations, a preliminary try of machine learning using block building programming (BBP) [29], a former version of the MBB algorithm, fails to work out the relationship functions of the triple-points coordinates with respect to the three key factors. Then the latest version of MBB algorithm [31] can learn the data and achieve a number of meaningful explicit formulas for $x_{\mathrm{A}}$ and $x_{\mathrm{B}}$ as listed in Tables 5 and 6, respectively.

A series of formulas for $x_{\mathrm{A}}$ and $x_{\mathrm{B}}$ were obtained by MBB machine learning algorithm with the corresponding coefficient of determination $R^{2}$. Here, $R^{2}$ is referred to as the goodness of fit and represented as a value between 0.0 and 1.0. A value of 1.0 indicates a perfect fit and the highest reliability of the correlation model. The output formulas for $x_{A}$ and $x_{B}$ with the top three $R^{2}$ are given in Tables 5 and 6, respectively. The formula in the first line of each table is rewritten as

$$
\begin{aligned}
x_{\mathrm{A}}= & -0.692693+5.01495 I r^{2}-0.819079 I r^{2} \sqrt{M a} \\
& -0.235192 \sqrt{\beta}-0.732974 I r^{2} \sqrt{\beta}, \\
x_{\mathrm{B}}= & -0.111626-2.37697 \sqrt{I r}+0.244051 M a \\
& -0.2628 M a \cos I r+0.00110349 \beta^{2} \cos I r .
\end{aligned}
$$

Using Eqs. (2) and (3) in Section 2.1 respectively, we can get the other coordinates of triple points $\mathrm{A}$ and B, i.e., $y_{\mathrm{A}}$ and $y_{\mathrm{B}}$.

\subsection{Precision evaluation}

The precision of the formulas worked out in Section 3.1 by using the MBB machine learning algorithm needs to be evaluated. Preliminarily, we can compare the locations of the triple points $A$ and $B$ respectively drew according to the formulas with the numerical results. The machine learning outputs and the numerical results are combined in Fig. 6 for a direct comparison. In each frame of the figure, the purple curves represent the machine learning outputs for the upper and the lower triple points which vary with Ir when $M a$ and $\beta$ are fixed. The overall wave configurations obtained by numerical simulations are depicted according to the Mach number contours of cases with different $I r$. It can be seen that the consistency is acceptable except that the deviation of the triple point A of case $I r=0.4$ (red) in Fig. 6 (a) is relatively large. The reason for such a deviation is that the interaction pattern is within the transitional region between type IV and type III. As mentioned in Section 3.1, the relationship functions between the coordinates of triple point A with Ir are different for different interaction types. Therefore, the deviation in the vicinity of the transitional region is a reflection of the underlying physics. Obviously, this multilevel block building algorithm can only output the continuous formulas and it is hard to avoid a little deviation within the transitional parameter region of the interaction types.

With the computational outputs of 225 cases and the machine learning formulas, a statistical analysis of the formula error is performed and listed in Table 7. As donated in Fig. 7 (a), $r$ means the distance of triple point $A$ to the cylinder center while $L$ is the standoff distance of the upper bow shock, i.e., $L=x_{\mathrm{A}}-R$. The operator $\delta$ indicates the difference between the two variables respectively obtained from the machine learning formula and the numerical simulation. In the table, the relative error of the standoff distance of the upper bow shock $\delta L / L$ is the largest and we used it to estimate the precision of the machine learning formula. Its maximum error is $13.16 \%$ while the average error is $2.72 \%$. The error distribution is given in Fig. 7 (b) by a sector diagram. Fortunately, we can find that over 80 percent of cases have relative errors lower than $5 \%$. On the other hand, only 2 percent of cases have relative errors larger than $10 \%$ which most likely fall in the transition parameter space of shock-shock interactions. Considering the unsteady nature of the shock-shock interactions, we can conclude that the precision of the machine learning formula is acceptable. 


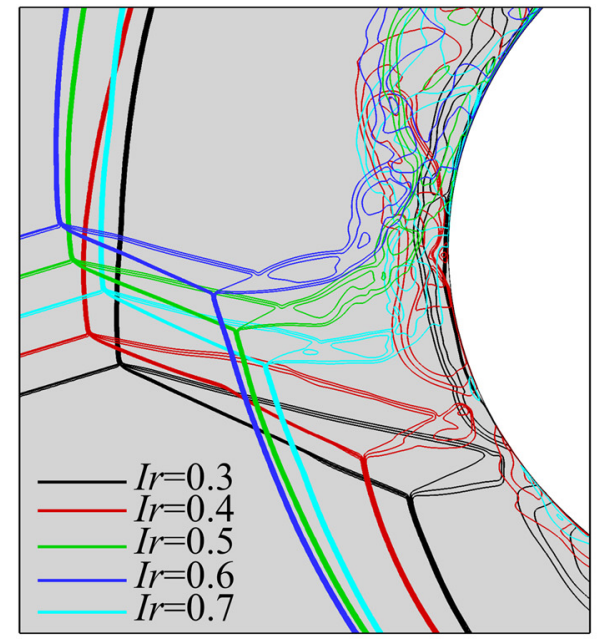

(a) $M a=7, \beta=19^{\circ}$

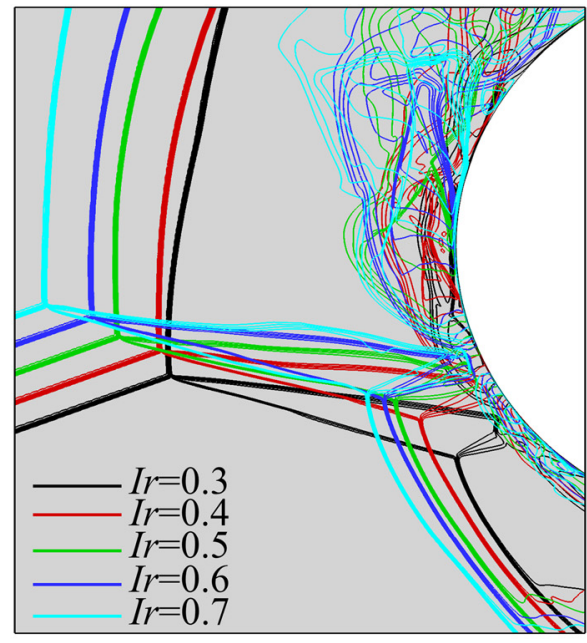

(b) $M a=9, \beta=22^{\circ}$

Fig. 5. The overall wave structures with different $I r$.

Table 5

Formulas for $x_{\mathrm{A}}$ achieved by MBB machine learning.

\begin{tabular}{|c|c|c|}
\hline Order & Formulas $x_{\mathrm{A}}=$ & $R^{2}$ \\
\hline 1 & $-0.692693+5.01495 I r^{2}-0.819079 I r^{2} \sqrt{M a}-0.235192 \sqrt{\beta}-0.732974 I r^{2} \sqrt{\beta}$ & 0.9569 \\
\hline 2 & $-1.84972+4.37143 \operatorname{Ir}+0.0306653 M a-0.141673 \operatorname{IrMa}-0.855294 \operatorname{Ir} \sqrt{\beta}$ & 0.9547 \\
\hline 3 & $7.18154-0.145304 I r^{2} M a-0.243041 \sqrt{\beta}-0.703906 I r^{2} \sqrt{\beta}-7.86041 \cos I r$ & 0.9544 \\
\hline$\ldots$ & $\cdots$ & $\ldots$ \\
\hline
\end{tabular}

Table 6

Formulas for $x_{\mathrm{B}}$ achieved by MBB machine learning.

\begin{tabular}{|c|c|c|}
\hline Order & Formulas $x_{\mathrm{B}}=$ & $R^{2}$ \\
\hline 1 & $-0.111626-2.37697 \sqrt{I r}+0.244051 M a-0.2628 M a \cos I r+0.00110349 \beta^{2} \cos I r$ & 0.9447 \\
\hline 2 & $-0.514993-2.75902 I r+1.2891 \sqrt{M a}+0.000949431 \beta^{2}-1.38203 \sqrt{M a} \cos I r$ & 0.9367 \\
\hline 3 & $0.301781-0.477088 \sqrt{M a}+0.884133 \operatorname{Ir} \sqrt{M a}+0.0003355 \sqrt{M a} \beta^{2}-3.89177 \sin I r$ & 0.9347 \\
\hline$\ldots$ & $\ldots$ & $\ldots$ \\
\hline
\end{tabular}

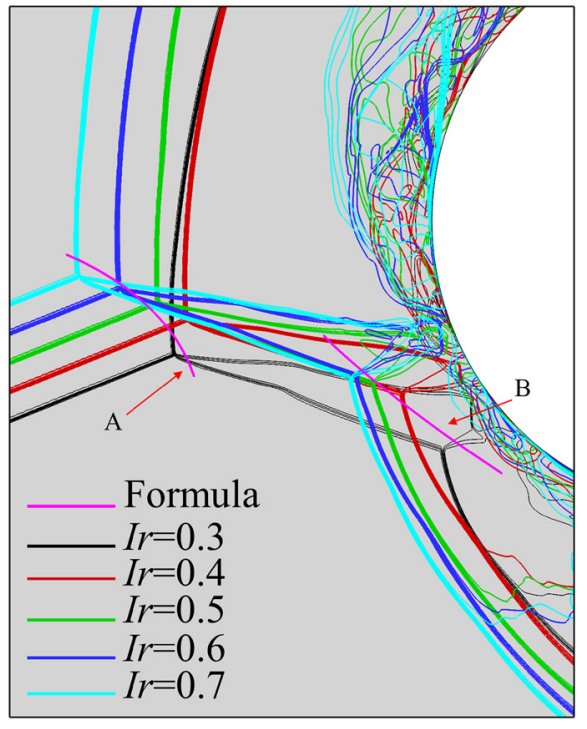

(a) $M a=8, \beta=22^{\circ}$

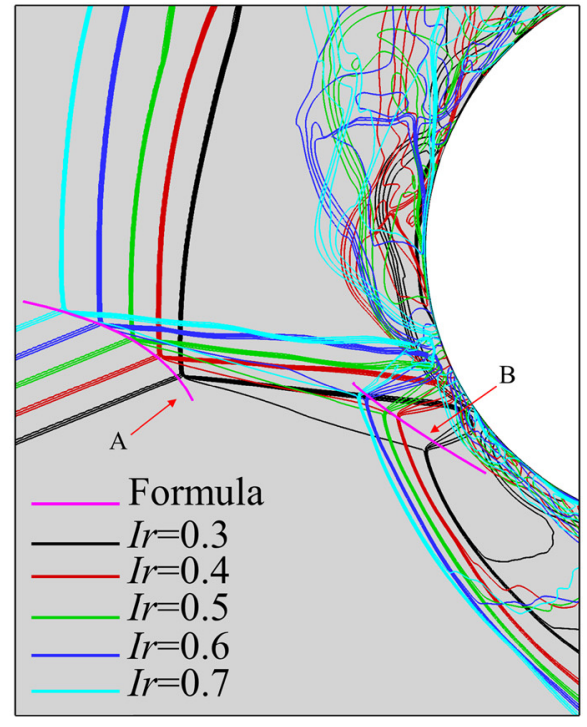

(b) $M a=10, \beta=22^{\circ}$

Fig. 6. The Mach number contours and the track of triple points with different Ir. 

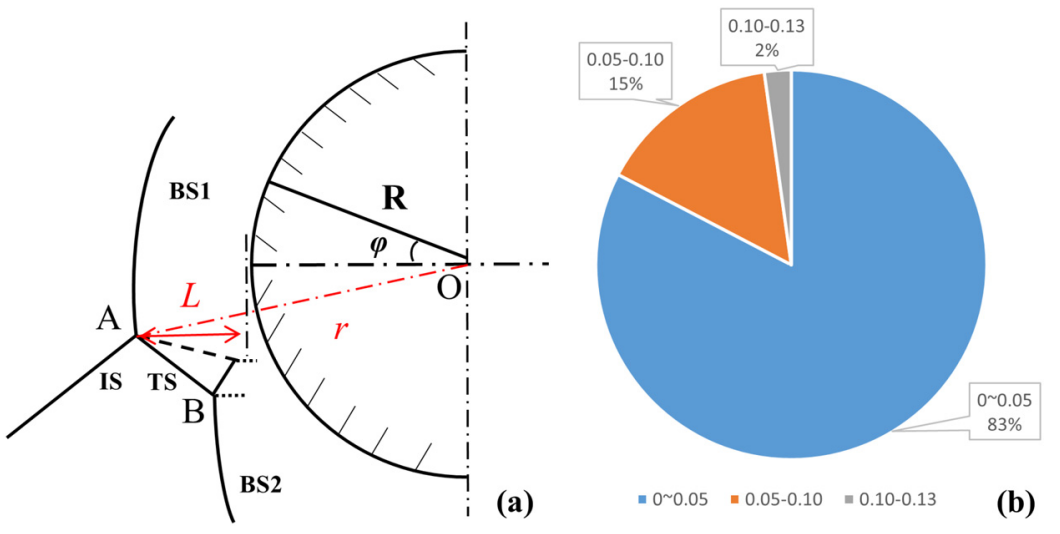

Fig. 7. The donation of $L$ and $r$ and the distribution of the relative error, $\delta L / L$.

Table 7

The statistical data for all cases.

\begin{tabular}{llllll}
\hline & $\delta x_{\mathrm{A}}(\delta L)$ & $\delta y_{\mathrm{A}}$ & $\delta r$ & $\delta r / r$ & $\delta L / L$ \\
\hline maximum & 0.0788 & 0.0386 & 0.0805 & 0.0487 & $\mathbf{0 . 1 3 1 6}$ \\
minimum & 0.0000 & 0.0000 & 0.0031 & 0.0017 & $\mathbf{0 . 0 0 0 0}$ \\
average & 0.0222 & 0.0108 & 0.0259 & 0.0141 & $\mathbf{0 . 0 2 7 2}$ \\
\hline
\end{tabular}

Table 8

The parameter range of interpolative cases.

\begin{tabular}{ll}
\hline Variables & Value \\
\hline Mach number, $M a$ & $6.5,7.5,8.5,9.5$ \\
Shock angle, $\beta /^{\circ}$ & $16.5,19.5,22.5$ \\
Location parameter, $I r$ & $0.35,0.45,0.55,0.65$ \\
\hline
\end{tabular}

Table 9

Error analysis using interpolative cases.

\begin{tabular}{llllll}
\hline & $\delta x_{\mathrm{A}}(\delta L)$ & $\delta y_{\mathrm{A}}$ & $\delta r$ & $\delta r / r$ & $\delta L / L$ \\
\hline maximum & 0.1054 & 0.0312 & 0.1099 & 0.0511 & $\mathbf{0 . 0 9 1 5}$ \\
minimum & 0.0003 & 0.0007 & 0.0015 & 0.0007 & $\mathbf{0 . 0 0 0 4}$ \\
average & 0.0187 & 0.0089 & 0.0214 & 0.0113 & $\mathbf{0 . 0 2 1 1}$ \\
\hline
\end{tabular}

Table 10

The parameter range of extrapolative cases.

\begin{tabular}{ll}
\hline Variables & Value \\
\hline Mach number, $M a$ & $5,11,12$ \\
Shock angle, $\beta /^{\circ}$ & 15,20 \\
Location parameter, $I r$ & $0.3,0.5$ \\
\hline
\end{tabular}

The aforementioned error analysis is based on the numerical data which are the machine learning samples in the meantime. The formula precision should be further evaluated by additional cases that are different from the samples already used. Therefore, further simulations containing $4 \times 3 \times 4=48$ cases as given in Table 8 by interpolating from the parameter space of the samples are conducted and the statistical error analysis using the output data of these cases is summarized in Table 9.

The maximum relative error of the bow shock standoff distance $\delta L / L$ is $9.15 \%$ while the average error is $2.11 \%$. It indicates that the machine learning formula of the triple point location is reasonable and reliable in the coverage of the samples.

Further simulations of $3 \times 2 \times 2=12$ cases where the parameters as listed in Table 10 go beyond the sample space are conducted to evaluate the extrapolation capability of machine learning formulas. The obtained maximum value of $\delta L / L$ is $7.93 \%$ and the average is $3.43 \%$ as summarized in Table 11 . Such an evaluation indicates that the formulas obtained by MBB machine learning al-
Table 11

Error analysis using extrapolative cases.

\begin{tabular}{llllll}
\hline & $\delta x_{\mathrm{A}}(\delta L)$ & $\delta y_{\mathrm{A}}$ & $\delta r$ & $\delta r / r$ & $\delta L / L$ \\
\hline maximum & 0.0801 & 0.0224 & 0.0817 & 0.0389 & $\mathbf{0 . 0 7 9 3}$ \\
minimum & 0.0006 & 0.0001 & 0.0049 & 0.0028 & $\mathbf{0 . 0 0 0 8}$ \\
average & 0.0277 & 0.0107 & 0.0320 & 0.0176 & $\mathbf{0 . 0 3 4 3}$ \\
\hline
\end{tabular}

gorithm are applicable and reliable with a certain extrapolation capability within the hypersonic regime.

\subsection{Prediction of the impinging location and the width of the jet flow}

The complex and unsteady characteristics of SSI give rise to great challenges in the design of hypersonic vehicles especially when aerodynamic heating is concerned. For the practical need during the initial phase of engineering design, the jet impinging location and the scale of the impinging spot are the key factors for the quick estimation of the maximum heating load and its location. The formulas obtained by MBB machine learning algorithm in the present work can be helpful and useful in solving such problems.

The formulas obtained by MBB can determine the locations of the triple points A and B. As analyzed in Section 2.1, with the inclinations of SL1 and the reflecting shock (BC) which can be theoretically calculated by the oblique shock theory, the intersecting location (point $\mathrm{C}$ ) of the shear layer and reflect shock can be further solved. In addition, the expansion wave emitting from point $\mathrm{C}$ and the successive reflected waves between the shear layers can be solved by the Prandtl-Mayer relation. Therefore, we have the coordinates of points A, B, and C and the inclinations of SL1 and SL2. The intersecting points of SL1 and SL2 with the cylinder surface identify the jet impinging location while the distance between the two points approximately defines the scale of jet impinging spot. The overall shock-shock interaction configuration solved by the MBB machine learning algorithm and the numerical results are combined in Fig. 8 for a direct comparison.

From Fig. 8, we can see that the machine learning method can predict the shock intersecting locations and the starting part of the jet for three types of shock-shock interactions with acceptable precisions. Fig. 8 (a) describes a type IV interaction in which the jet flow hits the wall perpendicularly. The predicted impinging location and width of the jet are both in agreement with the numerical results. In Fig. 8 (b), it is type IVa interaction in which the jet flow turns upward along the cylinder surface. As can be seen in the numerical contours, the unsteady interaction between the shear layer and the boundary layer makes the jet bend. Such a phenomenon is not taken into account in the machine learning and theoretical analysis, and thus the deviation in predicting SL2 


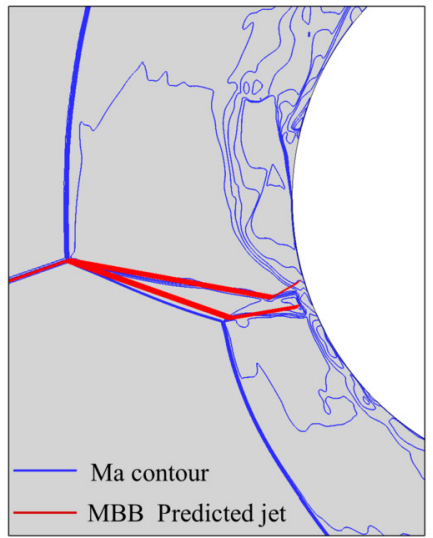

(a) $M a=9, \beta=19^{\circ}, I r=0.4$

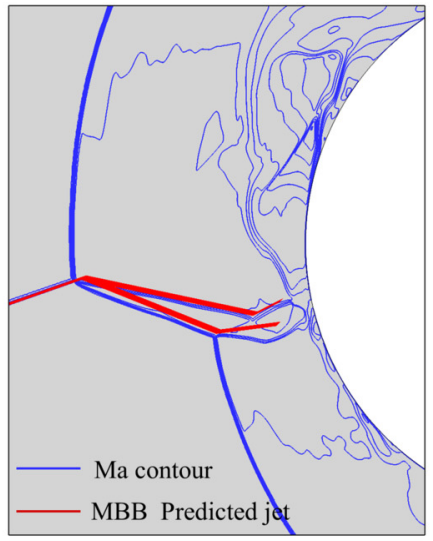

(b) $M a=8, \beta=19^{\circ}, I r=0.5$

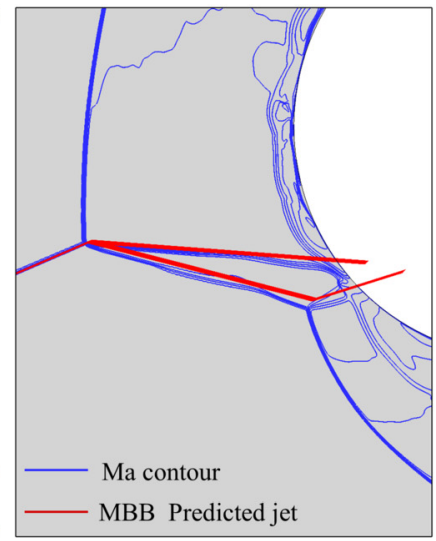

(c) $M a=9, \beta=23^{\circ}, I r=0.3$

Fig. 8. The prediction of the jet configuration.
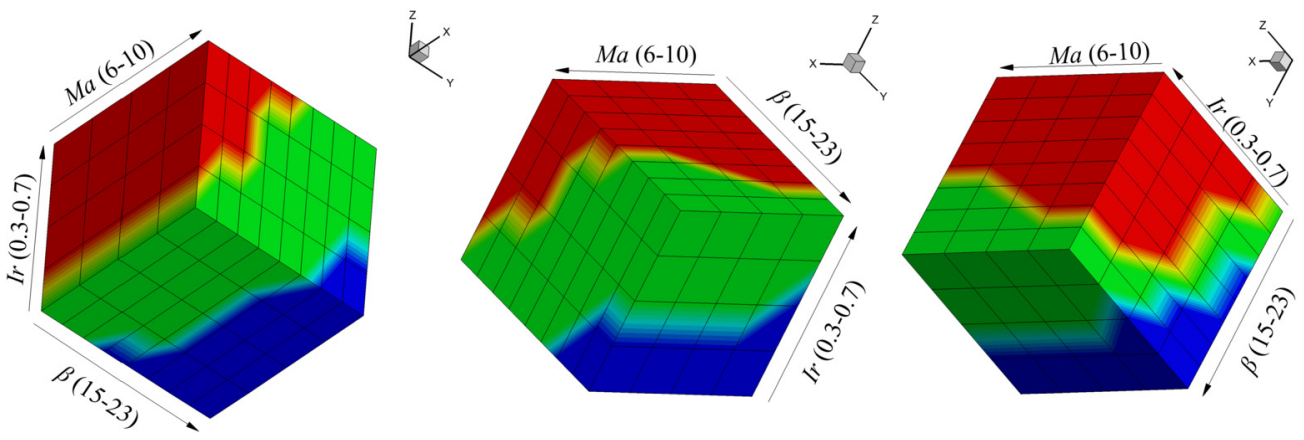

Fig. 9. The 3D distribution of the interaction types in the space of ( $M a, \beta$, and $I r)$.

in a type IVa interaction is reasonable. In Fig. 8 (c), it is a type III interaction in which the shear layer impinges on the surface. In summary, the machine learning method can predict the impinging location and jet width for type IV and III interactions with acceptable precisions. However, due to the unsteady interaction between the jet and the body surface, a slight deviation appears in the type IVa interaction.

\subsection{The transition criteria}

The shock-shock interaction type of each computed case is given in Table 4. Combining the interaction types and their corresponding input parameter, i.e., $M a, \beta$, and $I r$, we may construct a $3 \mathrm{D}$ distribution of the interaction type in the three-dimensional (3D) space of $(M a, \beta, I r)$ as depicted in Fig. 9. Here, the occupied space of type IVa, IV, and III interactions are represented by red, green, and blue respectively. It can be seen that the distribution space is divided into 3 parts by two 3D surfaces corresponding to the two transition criteria of IVa $\leftrightarrow$ IV and IV $\leftrightarrow$ III. For the data shown in Fig. 9 that are discrete points, we used the interpolation of boundary points to get the center surfaces of these two boundaries as the transitional criteria. The two surfaces of transitional criteria are shown in Fig. 10, which appear irregular and strong three-dimensional. With the MBB machine learning algorithm, we learned the approximate equations of the two transitional surfaces based on their coordinates. They can be respectively expressed as

$\sin \left(\left[\frac{M a-\beta}{\beta}\right]^{2}\right)-I r=0$

$\cos (\sin (\sqrt{M a+\beta}))-I r=0$.
Fig. 11 shows the transition criteria obtained by MBB machine learning algorithm. The red surface corresponds to Eq. (6) representing type IV $\leftrightarrow$ III transition criterion while the blue surface defined by Eq. (7) is for type IVa $\leftrightarrow$ IV transition criterion. When the three key factors $I r, M a$ and $\beta$ are given, according the Eq. (6) and Eq. (7), the space where the coordinate $(I r, M a, \beta)$ is located can be known. Then we can know which interaction type would occur. We can see from Fig. 11 that the two surfaces are approximately perpendicular to the Ir-axis, which means the transition criteria of interaction type are much more sensitive to the factor Ir than the other two. Moreover, the room between the two surfaces gets bigger with the increase of the Ma. That is to say, type IV interaction is more likely to appear at higher Mach numbers.

\subsection{Conclusions}

The overall wave configuration of a shock-shock interaction is hard to be theoretically solved through traditional shock theories due to its complexity. Such a solution, if available, is useful for the prediction of aerodynamic heating load during the initial design phase of a hypersonic vehicle. A machine learning algorithm, i.e., multilevel block building algorithm, is introduced into the present work to correlate the overall shock-shock interaction configuration with given flow parameters, such as $M a, \beta$, and $I r$. Here, the freestream flow Mach number $M a$, the incident shock angle $\beta$ of the oblique shock wave, and the geometric parameter Ir dominate the wave configuration of the shock-shock interaction, which was studied in the present work. The samples needed for machine learning are obtained by numerical simulations of 225 cases in the parameter space of $(M a, \beta, I r)$. Based on the numerical samples, the multilevel block building algorithm is used to learn the formulas of the triple-points' coordinates which define the overall wave 

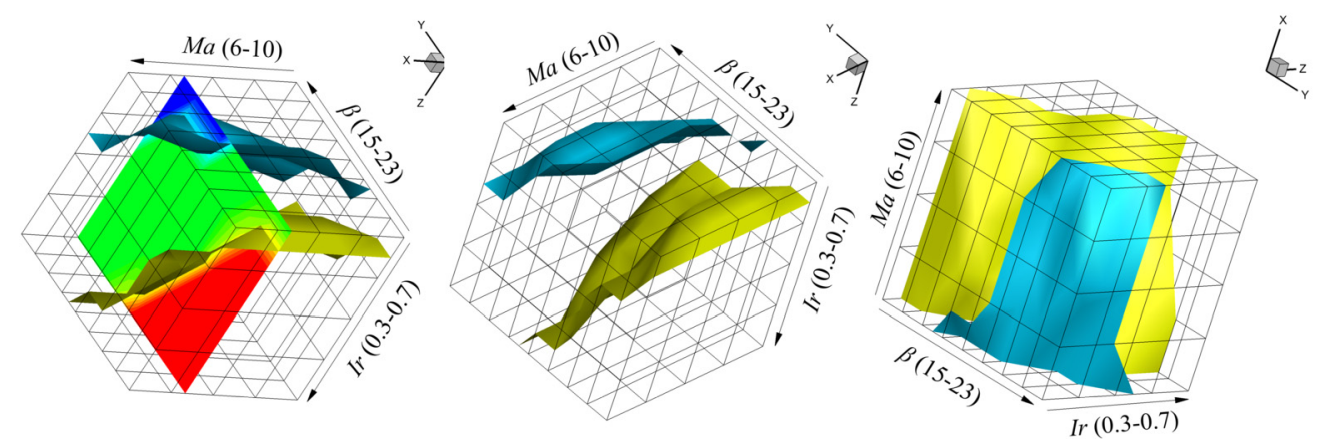

Fig. 10. The two 3D surfaces of the transition criteria.
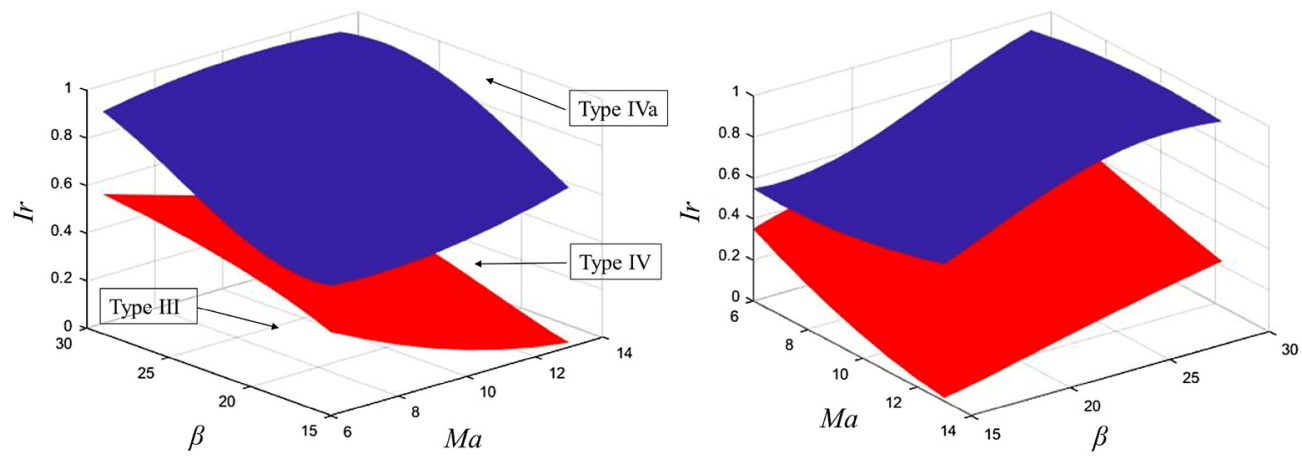

Fig. 11. The two faces of transition criteria obtained by MBB.

configuration of a shock-shock interaction. The formulas are further validated by using 48 numerical cases interpolated inside the sample parameter space and 12 cases extrapolated, respectively. The validation of the aforementioned formulas indicates perfect applicability and extrapolation capability for the shock-shock interactions in the present work.

With the coordinates of the triple points which are solved by multilevel block building algorithm and the flow parameters which can be solved by shock theories, the location of the jet impinging spot on the surface and its scale can be predicted. These two parameters obtained by the aforementioned machine learning based method are useful for the estimation of the peak value of heat flux induced by jet impingement. Tests indicate that the aforementioned machine learning based method works well for type IV interactions with perfect precision. On the other hand, due to the interaction of shear layers with the boundary layer along the surface, the deviation is relatively large for the type IVa interaction. However, the precision of this method for this type of interaction is still acceptable. Based on the numerical samples, what's more, the machine learning algorithm can achieve the approximate transition criteria in the parameter space of $(M a, \beta, I r)$ respectively for type IVa $\leftrightarrow$ IV transition and type IV $\leftrightarrow$ III transition. The result indicates that the transition criteria are much more sensitive to the parameter Ir than the other two and type IV interaction is more likely to appear at higher freestream flow Mach numbers.

The application of the multilevel block building algorithm in predicting the triple-points' coordinates of a shock-shock interaction indicates that the introduction of the machine learning into the traditional shock dynamics may bring new ideas and new methods to solve complex shock dynamic problems.

\section{Declaration of competing interest}

The authors declare that they have no known competing financial interests or personal relationships that could have appeared to influence the work reported in this paper.

\section{Acknowledgements}

This work was supported by the National Key Research and Development Plan of China under Grant No. 2019YFA0405204 and the National Natural Science Foundation of China under Grant No. 11672308 and 11532014.

\section{References}

[1] B.E. Edney, Effects of shock impingement on the heat transfer around blunt bodies, AIAA J. 6 (1968) 15-21.

[2] J.W. Keyes, F.D. Hains, Analytical and Experimental Studies of Shock Interference Heating in Hypersonic Flows, NASA Langley Research Center, Washington, United States, 1973.

[3] M. Holden, J. Moselle, A. Wieting, C. Glass, Studies of aerothermal loads generated in regions of shock/shock interaction in hypersonic flow, in: 26th Aerospace Sciences Meeting, Reno, Nevada, 1988, p. 477.

[4] A.R. Wieting, M.S. Holden, Experimental shock-wave interference heating on a cylinder at Mach 6 and 8, AIAA J. 27 (1989) 1557-1565.

[5] X. Zhong, Application of essentially nonoscillatory schemes to unsteady hypersonic shock-shock interference heating problems, AIAA J. 32 (1994) 1606-1616.

[6] Datta Gaitonde, J.S. Shang, On the structure of an unsteady type IV interaction at Mach 8, Comput. Fluids 24 (4) (1995) 469-485.

[7] C.A. Lind, M.J. Lewis, Unsteady characteristics of a hypersonic type IV shock interaction, J. Aircr. 32 (1995) 1286-1293.

[8] Y.B. Chu, X.Y. Lu, Characteristics of unsteady type IV shock/shock interaction, Shock Waves 22 (2012) 225-235.

[9] G.H. Furumoto, X. Zhong, J.C. Skiba, Numerical studies of real-gas effects on two-dimensional hypersonic shock-wave/boundary-layer interaction, Phys. Fluids 9 (1997) 191-210.

[10] Serge M. Boldyrev, VolfYa Borovoy, A.Y. Chinilov, A thorough experimental investigation of shock/shock interferences in high Mach number flows, Aerosp. Sci. Technol. 5 (2001) 167-178.

[11] F. Xiao, Z. Li, Y. Zhu, J. Yang, Hypersonic type-IV shock/shock interactions on a blunt body with forward-facing cavity, J. Spacecr. Rockets 54 (2017) 506-512.

[12] A. Khatta, J. Gopalan, Hypersonic shock tunnel studies of Edney type III and IV shock interactions, Aerosp. Sci. Technol. 72 (2018) 335-352.

[13] F. Grasso, C. Purpura, B. Chanetz, J. Délery, Type III and type IV shock/shock interferences: theoretical and experimental aspects, Aerosp. Sci. Technol. 7 (2003) 93-106.

[14] F.S. Billig, Shock-wave shapes around spherical- and cylindrical-nosed bodies, J. Spacecr. Rockets 4 (1967) 822-823. 
[15] M.J. Frame, M.J. Lewis, Analytical solution of the type IV shock interaction, J. Propuls. Power 13 (1997) 601-609.

[16] S. Allison, H. Bai, B. Jayaraman, Wind estimation using quadcopter motion: a machine learning approach, Aerosp. Sci. Technol. 98 (2020) 105699

[17] X. Yan, J. Zhu, M. Kuang, X. Wang, Aerodynamic shape optimization using a novel optimizer based on machine learning techniques, Aerosp. Sci. Technol. 86 (2019) 826-835.

[18] Y. Yu, H. Yao, Y. Liu, Aircraft dynamics simulation using a novel physics-based learning method, Aerosp. Sci. Technol. 87 (2019) 254-264.

[19] K. Boychev, G.N. Barakos, R. Steijl, Flow physics and sensitivity to RANS modelling assumptions of a multiple shock wave/turbulent boundary layer interaction, Aerosp. Sci. Technol. 97 (2020) 105640.

[20] V. Shinde, J. McNamara, D. Gaitonde, Control of transitional shock wave boundary layer interaction using structurally constrained surface morphing, Aerosp. Sci. Technol. 96 (2020) 105545.

[21] H.D. Lim, T.H. New, R. Mariani, Y.D. Cui, Effects of bevelled nozzles on standoff shocks in supersonic impinging jets, Aerosp. Sci. Technol. 94 (2019) 105371

[22] S.P. Nagdewe, G.R. Shevare, H.-D. Kim, Study on the numerical schemes for hypersonic flow simulation, Shock Waves 19 (2009) 433-442.

[23] B.J. McBride, M.J. Zehe, S. Gordon, NASA Glenn Coefficients for Calculating Thermodynamic Properties of Individual Species, NASA TP-2002-211556, 2002.

[24] Z. Zhang, K. Ma, W. Zhang, X. Han, Y. Liu, Z. Jiang, Numerical investigation of a Mach 9 oblique detonation engine with fuel pre-injection, Aerosp. Sci. Technol. 105 (2020) 106054.
[25] H. Lu, L. Yue, Y. Xiao, X. Zhang, Interaction of isentropic compression waves with a bow shock, AIAA J. 51 (2013) 2474-2484.

[26] N.N. Smirnov, V.B. Betelin, R.M. Shagaliev, V.F. Nikitin, I.M. Belyakov, Y.N. Deryuguin, S.V. Aksenov, D.A. Korchazhkin, Hydrogen fuel rocket engines simulation using LOGOS code, Int. J. Hydrog. Energy 39 (2014) 10748-10756.

[27] N.N. Smirnov, V.B. Betelin, V.F. Nikitin, L.I. Stamov, D.I. Altoukhov, Accumulation of errors in numerical simulations of chemically reacting gas dynamics, Acta Astronaut. 117 (2015) 338-355.

[28] V.V. Tyurenkova, L.I. Stamov, Flame propagation in weightlessness above the burning surface of material, Acta Astronaut. 159 (2019) 342-348.

[29] C. Chen, C. Luo, Z. Jiang, Block building programming for symbolic regression, Neurocomputing 275 (2018) 1973-1980.

[30] I. Arnaldo, U.-M. O’Reilly, K. Veeramachaneni, Building predictive models via feature synthesis, in: Proceedings of the 2015 Annual Conference on Genetic and Evolutionary Computation, 2015, pp. 983-990.

[31] C. Chen, C. Luo, Z. Jiang, A multilevel block building algorithm for fast modeling generalized separable systems, Expert Syst. Appl. 109 (2018) 25-34.

[32] K. Veeramachaneni, I. Arnaldo, O. Derby, U.-M. O’Reilly, FlexGP: cloud-based ensemble learning with genetic programming for large regression problems, J. Grid Comput. 13 (2015) 391-407.

[33] C. Luo, Z. Chen, Z. Jiang, Performance evaluation of symbolic regression methods on hypersonic aerodynamic data modeling, in: 32nd International Symposium on Shock Waves, Singapore, 2019. 